\title{
Average Value of the Euler Function on Binary Palindromes
}

by

\author{
William D. BANKS and Igor E. SHPARLINSKI
}

Presented by Andrzej SCHINZEL

Summary. We study values of the Euler function $\varphi(n)$ taken on binary palindromes of even length. In particular, if $\mathcal{B}_{2 \ell}$ denotes the set of binary palindromes with precisely $2 \ell$ binary digits, we derive an asymptotic formula for the average value of the Euler function on $\mathcal{B}_{2 \ell}$.

1. Introduction. Consider the binary representation of an arbitrary positive integer $n$ :

$$
n=\sum_{k=0}^{L-1} \beta_{k} \cdot 2^{k},
$$

where $\beta_{k} \in\{0,1\}$ for $k=0,1, \ldots, L-1$, and $\beta_{L-1}=1$. The integer $n$ is said to be a binary palindrome if its digits satisfy the symmetry relation

$$
\beta_{k}=\beta_{L-1-k} \quad \text { for } k=0,1, \ldots, L-1 .
$$

The number $L$ in (1) is called the length of $n$. Let $\mathcal{B}_{L}$ denote the set of all binary palindromes of length $L$.

In this paper, we obtain an asymptotic formula for the average value of the Euler function $\varphi(n)$ taken on binary palindromes of even length $L=2 \ell$; that is, we estimate sums of the form

$$
\mathcal{S}(\ell)=\frac{1}{\# \mathcal{B}_{2 \ell}} \sum_{n \in \mathcal{B}_{2 \ell}} \varphi(n)
$$

Our main result (Theorem 5) is obtained from estimates for sums of the

2000 Mathematics Subject Classification: Primary 11A63; Secondary 11N37.

Key words and phrases: Euler function, binary palindrome. 
form

$$
\mathcal{T}(\ell, r, s)=\frac{1}{\# \mathcal{B}_{2 \ell}} \sum_{n \in \mathcal{B}_{2 \ell}} \frac{\varphi(r n+s)}{r n+s}
$$

which are given in Theorem 3 below; this result may be of independent interest.

It has been shown in [1] that almost all palindromes (with respect to a fixed base $b \geq 2$ ) are composite, and several additional arithmetic properties of palindromes have been established in [3]. Our estimates for the sums $\mathcal{S}(\ell)$ and $\mathcal{T}(\ell, r, s)$ rely on asymptotic formulas and upper bounds from [1,3] for the number of palindromes lying in a fixed arithmetic progression.

Throughout the paper, implied constants in the symbols $O$ and $\ll$ are absolute. We recall that the notations $U=O(V)$ and $U \ll V$ are equivalent to the assertion that the inequality $|U| \leq c V$ holds for some constant $c>0$.

2. Congruences with palindromes. Let us define

$$
\mathcal{B}_{L}(a, q)=\left\{n \in \mathcal{B}_{L}: n \equiv a(\bmod q)\right\}
$$

LEMmA 1. Let $q \geq 1$ be squarefree and $\ell \geq 5+q^{2} \log q$. If $q$ and a are both even, or if $3 \mid q$ and $3 \nmid a$, then $\# \mathcal{B}_{2 \ell}(a, q)=0$; in all other cases, we have

$$
\# \mathcal{B}_{2 \ell}(a, q)=\frac{\# \mathcal{B}_{2 \ell} \cdot \operatorname{gcd}(q, 6)}{q}\left\{1+O\left(\exp \left(-\ell / q^{2}\right)\right)\right\}
$$

Proof. Since every palindrome $n \in \mathcal{B}_{2 \ell}$ is odd and divisible by 3 , it is clear that $\mathcal{B}_{2 \ell}(a, q)=\emptyset$ if $q$ and $a$ are both even, or if $3 \mid q$ and $3 \nmid a$; in all other cases, we have $\mathcal{B}_{2 \ell}(a, q)=\mathcal{B}_{2 \ell}(a, q / \operatorname{gcd}(q, 6))$, and the result follows immediately from [1, Proposition 4.2].

We also need a nontrivial bound for $\# \mathcal{B}_{2 \ell}(a, q)$ without any restrictions on the size or the arithmetic structure of $q$. For $a=0$ the following bound has been obtained in [3]; the proof, however, extends to the general case without any modifications.

LEMMA 2. Uniformly for integers $L \geq 1, q \geq 1$ and a, we have

$$
\# \mathcal{B}_{L}(a, q) \ll \frac{\# \mathcal{B}_{L}}{q^{1 / 2}} .
$$

3. Main results. We begin by deriving an asymptotic formula for the sum $\mathcal{T}(\ell, r, s)$ given by (3). To state the formula, we define the following 
constants:

$$
\begin{aligned}
& \alpha(r, s)=\left\{\begin{array}{ll}
3 / 4 & \text { if } 2 \mid r \text { and } 2 \mid s, \\
3 / 2 & \text { if } 2 \mid r \text { and } 2 \nmid s, \\
1 & \text { if } 2 \nmid r,
\end{array} \quad \gamma(s)= \begin{cases}1 & \text { if } s \equiv 0(\bmod 6), \\
3 / 4 & \text { if } s \equiv \pm 1(\bmod 6), \\
3 / 2 & \text { if } s \equiv \pm 2(\bmod 6), \\
1 / 2 & \text { if } s \equiv 3(\bmod 6),\end{cases} \right. \\
& \beta(r, s)=\left\{\begin{array}{ll}
4 / 3 & \text { if } 3 \mid r \text { and } 3 \mid s, \\
8 / 9 & \text { if } 3 \mid r \text { and } 3 \nmid s, \\
1 & \text { if } 3 \nmid r,
\end{array} \quad \zeta(2, r)=\prod_{p \mid r}\left(1-\frac{1}{p^{2}}\right)^{-1} .\right.
\end{aligned}
$$

THEOREM 3. Uniformly for integers $r \geq 1$ and $s \geq 0$, we have

$$
\mathcal{T}(\ell, r, s)=\frac{\varphi(t)}{t} \alpha(r, s) \beta(r, s) \gamma(s) \zeta(2, r) \cdot \frac{6}{\pi^{2}}+O\left((\ell / \log \ell)^{-1 / 4}\right),
$$

where $t=\operatorname{gcd}(r, s)$.

Proof. We can assume that $\ell$ is large since the result is trivial if $\ell$ is bounded.

For every positive integer $k$, we have

$$
\frac{\varphi(k)}{k}=\sum_{\delta \mid k} \frac{\mu(\delta)}{\delta},
$$

where $\mu(\delta)$ is the Möbius function; see (5.1) in [4, Chapter 1]. Therefore,

$$
\begin{aligned}
\mathcal{T}(\ell, r, s) & =\frac{1}{\# \mathcal{B}_{2 \ell}} \sum_{n \in \mathcal{B}_{2 \ell}} \sum_{\delta \mid(r n+s)} \frac{\mu(\delta)}{\delta} \\
& =\frac{1}{\# \mathcal{B}_{2 \ell}} \sum_{\delta \geq 1} \frac{\mu(\delta)}{\delta} \#\left\{n \in \mathcal{B}_{2 \ell}: \delta \mid(r n+s)\right\} .
\end{aligned}
$$

Recall that $t=\operatorname{gcd}(r, s)$. Every squarefree integer $\delta \geq 1$ can be expressed uniquely in the form $\delta=c d$, where $c \mid t$ and $\operatorname{gcd}(d, t)=1$. The following statements are equivalent:

(i) $\delta \mid(r n+s)$;

(ii) $d \mid(r n+s)$;

(iii) $\operatorname{gcd}(d, r)=1$ and $n \equiv-r^{-1} s(\bmod d)$.

Indeed, (i) $\Leftrightarrow($ ii) since $c \mid(r n+s)$ and $\operatorname{gcd}(c, d)=1$. Next, suppose (ii) holds. If a prime $p$ divides $\operatorname{gcd}(d, r)$, then $p$ also divides $s$, which contradicts the fact that $\operatorname{gcd}(d, t)=1$; therefore, $\operatorname{gcd}(d, r)=1$, and the implication (ii) $\Rightarrow$ (iii) is clear. The implication (iii) $\Rightarrow$ (ii) is obvious. 
Since $\mu(\delta) / \delta$ is multiplicative, we therefore have

$$
\begin{aligned}
\mathcal{T}(\ell, r, s) & =\frac{1}{\# \mathcal{B}_{2 \ell}} \sum_{c \mid t} \frac{\mu(c)}{c} \sum_{\substack{d \geq 1 \\
\operatorname{gcd}(d, t)=1}} \frac{\mu(d)}{d} \#\left\{n \in \mathcal{B}_{2 \ell}: \delta \mid(r n+s)\right\} \\
& =\frac{\varphi(t)}{t} \frac{1}{\# \mathcal{B}_{2 \ell}} \sum_{\substack{d \geq 1 \\
\operatorname{gcd}(d, r)=1}} \frac{\mu(d)}{d} \# \mathcal{B}_{2 \ell}\left(a_{d}, d\right),
\end{aligned}
$$

where each $a_{d}$ satisfies the congruence $a_{d} \equiv-r^{-1} s(\bmod d)$. Now put

$$
D=\lfloor\sqrt{\ell / 2 \log \ell}\rfloor,
$$

and write

$$
\mathcal{T}(\ell, r, s)=\frac{\varphi(t)}{t}\left(\Sigma_{1}+\Sigma_{2}\right)
$$

where

$$
\begin{aligned}
& \Sigma_{1}=\sum_{\substack{1 \leq d \leq D \\
\operatorname{gcd}(d, r)=1}} \frac{\mu(d)}{d} \frac{\# \mathcal{B}_{2 \ell}\left(a_{d}, d\right)}{\# \mathcal{B}_{2 \ell}}, \\
& \Sigma_{2}=\sum_{\substack{d>D \\
\operatorname{gcd}(d, r)=1}} \frac{\mu(d)}{d} \frac{\# \mathcal{B}_{2 \ell}\left(a_{d}, d\right)}{\# \mathcal{B}_{2 \ell}} .
\end{aligned}
$$

Since $\ell \geq 5+D^{2} \log D$ once $\ell$ is sufficiently large, we can apply Lemma 1 to the sum $\Sigma_{1}$. Defining

we obtain

$$
\theta_{s}(d)= \begin{cases}0 & \text { if } d \text { and } s \text { are both even, or } 3 \mid d \text { and } 3 \nmid s, \\ 1 & \text { otherwise }\end{cases}
$$

$$
\begin{aligned}
\Sigma_{1} & =\sum_{\substack{1 \leq d \leq D \\
\operatorname{gcd}(d, r)=1}} \frac{\mu(d) \theta_{s}(d) \operatorname{gcd}(d, 6)}{d^{2}}+O\left(\sum_{1 \leq d \leq D} \frac{\exp \left(-\ell / d^{2}\right)}{d^{2}}\right) \\
& =\sum_{\substack{d \geq 1 \\
\operatorname{gcd}(d, r)=1}} \frac{\mu(d) \theta_{s}(d) \operatorname{gcd}(d, 6)}{d^{2}}+O\left(D^{-1}+\exp \left(-\ell / D^{2}\right)\right) .
\end{aligned}
$$

Since $\theta_{s}(d)$ is multiplicative for every integer $s$, it follows that

$$
\begin{aligned}
\sum_{\substack{d \geq 1 \\
\operatorname{gcd}(d, r)=1}} \frac{\mu(d) \theta_{s}(d) \operatorname{gcd}(d, 6)}{d^{2}}=\prod_{p \nmid r}\left(1-\frac{\theta_{s}(p) \operatorname{gcd}(p, 6)}{p^{2}}\right) \\
=\prod_{p \mid r}\left(1-\frac{\theta_{s}(p) \operatorname{gcd}(p, 6)}{p^{2}}\right)^{-1} \prod_{p}\left(1-\frac{\theta_{s}(p) \operatorname{gcd}(p, 6)}{p^{2}}\right) .
\end{aligned}
$$

It is easily verified on a case-by-case basis that 


$$
\begin{aligned}
\prod_{p \mid r}\left(1-\frac{\theta_{s}(p) \operatorname{gcd}(p, 6)}{p^{2}}\right)^{-1} & =\alpha(r, s) \beta(r, s) \zeta(2, r) \\
\prod_{p}\left(1-\frac{\theta_{s}(p) \operatorname{gcd}(p, 6)}{p^{2}}\right) & =\gamma(s) \prod_{p}\left(1-\frac{1}{p^{2}}\right)=\gamma(s) \frac{6}{\pi^{2}} .
\end{aligned}
$$

Therefore,

$$
\Sigma_{1}=\alpha(r, s) \beta(r, s) \gamma(s) \zeta(2, r) \cdot \frac{6}{\pi^{2}}+O\left(D^{-1}+\exp \left(-\ell / D^{2}\right)\right) .
$$

Using Lemma 2 to bound the sum $\Sigma_{2}$, we also have

$$
\Sigma_{2} \ll \sum_{d>D}^{\infty} d^{-3 / 2} \ll D^{-1 / 2} .
$$

Combining this bound with the estimate (6), it follows that

$$
\mathcal{T}(\ell, r, s)=\frac{\varphi(t)}{t} \alpha(r, s) \beta(r, s) \gamma(s) \zeta(2, r) \cdot \frac{6}{\pi^{2}}+O\left(D^{-1 / 2}+\exp \left(-\ell / D^{2}\right)\right) .
$$

Recalling the choice of $D$ given by (5), we obtain the stated result.

As the special case $r=1$ and $s=0$ of Theorem 3, we have:

Corollary 4. The following estimate holds as $\ell \rightarrow \infty$ :

$$
\frac{1}{\# \mathcal{B}_{2 \ell}} \sum_{n \in \mathcal{B}_{2 \ell}} \frac{\varphi(n)}{n}=\frac{6}{\pi^{2}}+O\left((\ell / \log \ell)^{-1 / 4}\right) .
$$

Using Theorem 3, we now derive an asymptotic formula for the sum $\mathcal{S}(\ell)$ defined by $(2)$.

THEOREM 5. The following estimate holds as $\ell \rightarrow \infty$ :

$$
S(\ell)=3 \cdot 2^{2 \ell-2}\left\{\frac{6}{\pi^{2}}+O\left((\ell / \log \ell)^{-1 / 4}\right)\right\} .
$$

Proof. Let $k$ be an integer such that $1 \leq k \leq \ell-1$. Observe the digits of an arbitrary palindrome $n \in \mathcal{B}_{2 \ell}$ follow the basic pattern:

\begin{tabular}{|l|l|l|l|l|}
\hline$\leftarrow a \rightarrow$ & $0 \cdots 0$ & $\leftarrow b \rightarrow$ & $0 \cdots 0$ & $\leftarrow \widehat{a} \rightarrow$ \\
\hline
\end{tabular}

with $i+j+k=\ell$. That is, every $n \in \mathcal{B}_{2 \ell}$ can be expressed in the form

$$
n=2^{2 \ell-k} a+2^{\ell-i} b+\widehat{a},
$$

where the integers $a, \widehat{a}, b, i$ have the following properties:

(i) $2^{k-1} \leq a<2^{k}$;

(ii) $\widehat{a}$ is uniquely determined by the condition $2^{2 \ell-k} a+\widehat{a} \in \mathcal{B}_{2 \ell}$;

(iii) $0 \leq i \leq \ell-k$;

(iv) $b=0$ if $i=0$, and $b \in \mathcal{B}_{2 i}$ otherwise. 
For convenience, let us define $\mathcal{B}_{0}=\{0\}$. Then, using (7), we have

$$
\mathcal{S}(\ell)=\frac{1}{\# \mathcal{B}_{2 \ell}} \sum_{a=2^{k-1}}^{2^{k}-1} \sum_{i=0}^{\ell-k} \sum_{b \in \mathcal{B}_{2 i}} \varphi\left(2^{2 \ell-k} a+2^{\ell-i} b+\widehat{a}\right) .
$$

Clearly,

$$
2^{2 \ell-k} a+2^{\ell-i} b+\widehat{a}=2^{2 \ell-k}(a+O(1)),
$$

and thus

$$
\mathcal{S}(\ell)=\frac{2^{2 \ell-k}}{\# \mathcal{B}_{2 \ell}} \sum_{a=2^{k-1}}^{2^{k}-1}(a+O(1)) \sum_{i=0}^{\ell-k} \sum_{b \in \mathcal{B}_{2 i}} \frac{\varphi\left(2^{2 \ell-k} a+2^{\ell-i} b+\widehat{a}\right)}{2^{2 \ell-k} a+2^{\ell-i} b+\widehat{a}} .
$$

To each inner sum, we apply Theorem 3 with $r=2^{\ell-i}$ and $s=2^{2 \ell-k} a+\widehat{a}$. Since $r \geq 2$ is a power of two, and $s \equiv 3(\bmod 6)$, we have

$$
\alpha(r, s)=3 / 2, \quad \beta(r, s)=1, \quad \gamma(s)=1 / 2, \quad \zeta(2, r)=4 / 3 .
$$

Therefore, taking into account the fact that $\# \mathcal{B}_{0}=1$ and $\# \mathcal{B}_{2 i}=2^{i-1}$ for all $i \geq 1$, and choosing $k=\lfloor\ell / 2\rfloor$, Theorem 3 implies that

$$
\begin{aligned}
\sum_{i=0}^{\ell-k} \sum_{b \in \mathcal{B}_{2 i}} \frac{\varphi\left(2^{2 \ell-k} a+2^{\ell-i} b+\widehat{a}\right)}{2^{2 \ell-k} a+2^{\ell-i} b+\widehat{a}} & =\sum_{i=0}^{\ell-k} \# \mathcal{B}_{2 i}\left\{\frac{6}{\pi^{2}}+O\left(\left(\frac{\log (i+2)}{i+1}\right)^{1 / 4}\right)\right\} \\
& =2^{\ell-k}\left\{\frac{6}{\pi^{2}}+O\left((\ell / \log \ell)^{-1 / 4}\right)\right\} .
\end{aligned}
$$

Substituting this estimate into (8), and noting that $\# \mathcal{B}_{2 \ell}=2^{\ell-1}$, it follows that

$$
\mathcal{S}(\ell)=2^{2 \ell-2 k+1}\left\{\frac{6}{\pi^{2}}+O\left((\ell / \log \ell)^{-1 / 4}\right)\right\} \sum_{a=2^{k-1}}^{2^{k}-1}(a+O(1)) .
$$

Computing the sum over $a$, and taking into account our choice $k=\lfloor\ell / 2\rfloor$, the result follows.

4. Remarks. The statement of Theorem 3 holds for all integers $r, s$ provided that $r n+s \geq 1$ for all $n \in \mathcal{B}_{2 \ell}$; this condition is needed to ensure that the sum (3) is defined.

It is easy to see that the average value of the palindromes in $\mathcal{B}_{2 \ell}$ is

$$
\frac{1}{\# \mathcal{B}_{2 \ell}} \sum_{n \in \mathcal{B}_{2 \ell}} n=3 \cdot 2^{2 \ell-2},
$$

hence Theorem 5 suggests that $\varphi(n)$ has the expected value $6 n / \pi^{2}$ for a "random" binary palindrome $n$ of even length.

It would be interesting to extend the results of this paper to palindromes of odd length, and also to have a "continuous" version of our results, that 
is, to estimate sums of the form

$$
\frac{1}{\# \mathcal{B}(x)} \sum_{n \in \mathcal{B}(x)} \varphi(n) \text { and } \frac{1}{\# \mathcal{B}(x)} \sum_{n \in \mathcal{B}(x)} \frac{\varphi(r n+s)}{r n+s},
$$

where $\mathcal{B}(x)$ denotes the set of all binary palindromes $n \leq x$. One can also consider such questions for palindromes in other bases.

It seems likely that the methods of this paper can be extended to obtain similar results for the sum of divisors function and for other functions that satisfy an appropriate analogue of (4). Nevertheless, we expect that for many other arithmetical functions, the problem of computing the average value of the function over a set of palindromes is notoriously difficult.

For a given set $\mathcal{A}$ of positive integers, if the average value of the Euler function on $\mathcal{A} \cap[1, x]$ can be determined, it is often possible to evaluate the asymptotic probability that two random elements in $\mathcal{A}$ are coprime, as well as the asymptotic probability that a random element in $\mathcal{A}$ is squarefree. For example, in [2] all three questions are treated in similar ways for sets of integers whose $g$-ary representations in base $g \geq 2$ are restricted in various ways. However, in the case of palindromes, the last two questions appear to be much more difficult than the first; the problems of finding asymptotic formulas for the sums

$$
\frac{1}{\left(\# \mathcal{B}_{2 \ell}\right)^{2}} \sum_{\substack{n, m \in \mathcal{B}_{2 \ell} \\ \operatorname{gcd}(n, m)=1}} 1 \text { and } \frac{1}{\# \mathcal{B}_{2 \ell}} \sum_{\substack{n \in \mathcal{B}_{2 \ell} \\ n \text { squarefree }}} 1
$$

remain open (with getting an improvement of Lemma 2 as the bottleneck).

\section{References}

[1] W. Banks, D. Hart and M. Sakata, Almost all palindromes are composite, Math. Res. Lett. 11 (2004), 853-868.

[2] W. Banks and I. Shparlinski, Arithmetic properties of numbers with restricted digits, Acta Arith. 112 (2004), 313-332.

[3] —, - Prime divisors of palindromes, Period. Math. Hungar. 51 (2005), 1-10.

[4] K. Prachar, Primzahlverteilung, Springer, Berlin, 1957.

William D. Banks

Department of Mathematics

University of Missouri

Columbia, MO 65211, U.S.A.

E-mail: bbanks@math.missouri.edu
Igor E. Shparlinski Department of Computing

Macquarie University Sydney, NSW 2109, Australia

E-mail: igor@ics.mq.edu.au

Received July 21, 2006;

received in final form September 1, 2006 